\title{
Polyamines in foods: development of a food database
}

\author{
Mohamed Atiya Ali ${ }^{1,3 *}$, Eric Poortvliet', Roger Strömberg ${ }^{2}$ and \\ Agneta Yngve 1,3
}

'Group for Public Health Nutrition, Department of Biosciences and Nutrition, Karolinska Institutet, Huddinge, Sweden;

${ }^{2}$ Department of Biosciences and Nutrition, NOVUM, Karolinska Institutet, Huddinge, Sweden; ${ }^{3}$ Faculty of Health,

Nutrition and Management, Akershus University College, Lillestrøm, Norway

\section{Abstract}

Background: Knowing the levels of polyamines (putrescine, spermidine, and spermine) in different foods is of interest due to the association of these bioactive nutrients to health and diseases. There is a lack of relevant information on their contents in foods.

Objective: To develop a food polyamine database from published data by which polyamine intake and food contribution to this intake can be estimated, and to determine the levels of polyamines in Swedish dairy products.

Design: Extensive literature search and laboratory analysis of selected Swedish dairy products. Polyamine contents in foods were collected using an extensive literature search of databases. Polyamines in different types of Swedish dairy products (milk with different fat percentages, yogurt, cheeses, and sour milk) were determined using high performance liquid chromatography (HPLC) equipped with a UV detector.

Results: Fruits and cheese were the highest sources of putrescine, while vegetables and meat products were found to be rich in spermidine and spermine, respectively. The content of polyamines in cheese varied considerably between studies. In analyzed Swedish dairy products, matured cheese had the highest total polyamine contents with values of $52.3,1.2$, and $2.6 \mathrm{mg} / \mathrm{kg}$ for putrescine, spermidine, and spermine, respectively. Low fat milk had higher putrescine and spermidine, 1.2 and $1.0 \mathrm{mg} / \mathrm{kg}$, respectively, than the other types of milk.

Conclusions: The database aids other researchers in their quest for information regarding polyamine intake from foods. Connecting the polyamine contents in food with the Swedish Food Database allows for estimation of polyamine contents per portion.

Keywords: putrescine; spermidine; spermine; polyamine contents; food polyamines

Received: 27 August 2010; Revised: 3 November 20 I0; Accepted: I4 December 20 I0; Published: I4 January 20II

$\mathrm{T}$ he Swedish Food Database provides regularly updated information on the nutritional composition for more than 2,000 foods and dishes, mostly Swedish representative foods (1). For each type of food, data on 52 nutrients are presented in terms of amounts per grams or portions of foods, in addition to some information on analytical methods and calculations, and are all available on the database. However, there is still a lack of information on the contents of some bioactive components in food, for example, polyamines.

Polyamines (putrescine, spermine, and spermidine) are aliphatic amines that are reported to be essential components of all living cells (2-4). Because of their roles in cellular growth, normal function, and proliferation, these biologically active compounds have been of main interest among the group of biogenic amines $(5,6)$. In addition, polyamines are involved in the differentiation of immune cells as well as in regulation of inflammatory reactions $(7,8)$, and they exert a suppressor effect on pulmonary immunologic and intestinal immunoallergic responses (9). In children, high polyamine intake during the first year has been significantly correlated to food allergy prevention (10). In addition, spermine and spermidine have been shown to display a significant antiglycation effect at physiological concentration suggesting new role for polyamines in diabetes (11).

The body pool of polyamines is maintained by three sources: endogenous or de novo biosynthesis, intestinal microorganisms, and exogenous supply through the diet $(5,12,13)$. This external dietary source provides a larger 
quantity of polyamines than the endogenous biosynthesis. The latter produces only about $1-2$ nmol of putrescine per hour per gram of tissue in the most active organs (14). Diet can to a certain extent regulate biosynthesis of polyamines (14). Thus, dietary polyamines have several important roles to play in this regard; supporting a normal metabolism (15) and maintaining optimal health as well as regulating the intracellular polyamine synthesis $(2,14,16,17)$. These seem to be of importance for maintaining the normal growth, maturation of the intestinal tract $(18,19)$. Since the level of polyamines decreases with age in animal organs (brain, kidney, spleen, and pancreas) (20), it has been suggested that maintenance of polyamine level from the diet is important to keep the functioning of various organs in the elderly (17). On the other hand, the cell growth promoting effect may also be negative in relation to cancer development. It has been shown that increased polyamine levels are associated with increased cell proliferation as well as expression of genes affecting tumor invasion and metastasis (21).

Polyamines are present in variable amounts in various foods (22). They originate from amino acids such as arginine and ornithine, which act as precursors and undergo decarboxylation processes by the action of putrefactive bacteria $(23,24)$. This explains why higher concentrations of polyamines are found in fermented food products such as sauerkraut, some sausages, and cheeses $(22,23)$. In plants, exposure to salt or osmotic stress seemed to enhance polyamine biosynthesis and, thereby, the contents of polyamines increased (25).

In mammalian cells, the ornithine forms putrescine by the action of the enzyme ornithine decarboxylase. Putrescine then forms spermidine and spermine through a reversible metabolic process (13).

The mean dietary intake of polyamines has been estimated in some countries such as Japan (6), the US (26), and the UK (2). The estimated values for the daily polyamine intake in different studies vary between 250 to $550 \mu \mathrm{mol}(6,22,26)$. In Europe, a higher intake of total polyamines $(700 \mu \mathrm{mol} / \mathrm{d})$ was reported in the Mediterranean regions (14) compared to $350-500 \mu \mathrm{mol} / \mathrm{d}$ in the UK and northern Europe (27). These dietary differences in the pattern of foods and polyamine intake have been associated with differences in the incidence of chronic diseases - the Mediterranean diet is known to be protective (14). The antioxidant effect of polyamines could play a major role in prevention from chronic diseases (28).

Studies reporting the contents of polyamines in foods $(2,4,16,29-34)$ show considerable variation in each food item between studies and even between samples of the same types of foods within studies.

The aim of this study was to develop a database of polyamine content in foods coupled with the Swedish
Food Database, based on literature research and completing analyses of typical Swedish dairy products.

\section{Methods}

\section{Database development and definition}

An extensive literature search of databases was conducted using the keywords polyamines, food, food analysis, diet, and the individual names for the three polyamines selected for addition to the database: putrescine, spermine, and spermidine. This search was done using PubMed, web of Science, and SciFinder Scholar that cover both Medline and CAPLUS databases. Both Medical Subject Headings (MeSH) and text word searching were used to locate any published laboratory data for polyamines contents in foods. Papers published between 1986 and 2009 were selected.

When a single reference reported multiple analyses for the same food group, or if values for an individual food item were obtained from several references, the mean for all given values was calculated. However, when values reported from different references were extremely different, the median was taken to minimize the influence of extreme values. The average polyamine content for each food was then entered in the Dietist XP. All collected information on polyamine content in different foods was gathered into an Excel file where they were classified into food groups.

Values for polyamines content in some of the studies have been reported in nmol/g of food. These values were converted into $\mathrm{mg} / \mathrm{kg}$ based on the appropriate equation (mol=mass/molecular weight). The molecular weights for putrescine, spermine, and spermidine are $88.15 \mathrm{~g} / \mathrm{mol}$, $145.25 \mathrm{~g} / \mathrm{mol}$, and $202.34 \mathrm{~g} / \mathrm{mol}$, respectively. In addition, portion size was also considered due to the fact that portion size and frequency of consumption of these foods vary considerably, which means that a normal food frequency questionnaire would not necessarily detect intake of products with high amounts of polyamines; for example, sauerkraut or well-fermented cheese (26).

\section{Portion size estimation}

The Swedish National Food Administration (Livsmedelsverket) has estimated the portion size for 1,334 foods. All portions in grams are available in the Swedish Food Database where the units in grams or deciliters can be found for most of the food available.

In the Dietist XP software (version 3.0, 2007) where the polyamine contents are entered, the weight of the food per portion size, the weight in grams per deciliter for liquid food, and the amounts in grams for each spoon or cup are all collected from the same data that is available in the Swedish Food Database from Livsmedelsverket, 2001. Thereby the content of each polyamine in foods per 
portion can also be estimated using the Dietist XP software.

\section{Sampling}

All samples were collected and analyzed between October and December 2009. Samples of cow's milk $(0.5 \%, 1.5 \%$, $3 \%$, and $4.5 \%$ fat), yogurt, and sour milk were purchased from the local market 1 day before the analysis and at least 1 day after production. The best before dates were in 7 days for milk, 4 and 2 weeks for yogurt and sour milk, respectively. Different types of cheese with low and high fat content, varied between short- and long-term storage were also purchased (at least 2 months before best before date). The brands of all these products were representative for the Swedish mostly purchased dairy products, with the exception of Gamle Ole, the Danish cheese that was included in the analysis due to its long fermentation period (52 weeks). Only 4.5\% fat milk and mature cheese were purchased from a different local market. Each sample was kept in the refrigerator at $4{ }^{\circ} \mathrm{C}$ until the next day, which was the same degree of refrigerating at the market. For each dairy product, five samples (packages) of the same type of product were subjected to replicate analysis.

\section{Chemicals}

Polyamine standards (putrescine, spermidine, and spermine), internal standard (1,7-diaminoheptane), perchloric acid 70\%, benzoyl chloride 99\%, and acetonitrile 99.9\% analytical grade were all purchased from SigmaAldrich (Chemie GmbH, Germany). Diethyl ether was obtained from Scharlau Chemie S.A, Spain.

\section{Analytical method}

For milk and yogurt, polyamine extraction was done by adding $3 \mathrm{ml}$ of $0.6 \mathrm{~N}$ perchloric acid to $5 \mathrm{ml}$ of the sample, spiked with $2 \mu \mathrm{M}$ internal standard (1,7diaminoheptane). For all types of cheese, $10 \mathrm{~g}$ of the sample was also spiked with a known amount of internal standard before the mixture was homogenized as described by Novella-Rodriguez et al. (33). The mixture was kept at $4^{\circ} \mathrm{C}$ for $1 \mathrm{~h}$ before centrifugation at $30,000 \mathrm{~g}$ $10 \mathrm{~min}$ at $4^{\circ} \mathrm{C}$ to separate the protein phase from the acidic extract phase including the polyamines. As previously detailed in methods for determination of polyamines using precolumn derivatization (35), the polyamine derivatization procedure was done by treating the acidic extract of polyamines with small amounts of $2 \mathrm{~N} \mathrm{NaOH}$ to keep the $\mathrm{pH}$ above 13. The mixture was then subjected to the derivatization procedure by adding $10 \mu \mathrm{l}$ of benzoyl chloride prior to polyamine extraction by diethyl ether (twice) and concentration by evaporation with nitrogen gas. The residue (benzoylated polyamines) was dissolved in $1 \mathrm{ml}$ of $38 \%$ acetonitrile in water (the same solvent that was used as mobile phase). The resulting solution was then filtered using a syringe filter (GF Millipore MA, USA) and an aliquot of $50 \mu \mathrm{l}$ of each sample was automatically injected onto a High Performance Liquid Chromatography (HPLC, Waters 2690) equipped with a Nova-Pak $\mathrm{C}_{18}$ column $(15 \times 3.9 \mathrm{~mm})$ and Waters UV detector 996. Data acquisition was accomplished with Millennium ${ }^{32}$ Version 3.0 system. The entire HPLC run was under isocratic elution with a flow rate of $1 \mathrm{ml} / \mathrm{min}$.

Benzoylated polyamines were detected by UV absorption at $198 \mathrm{~nm}$, as it has been shown to increase the absorbance by ca. 50 times when acetonitrile is used as solvent (36). Polyamine identification was based on comparison between the retention times of polyamine standards. These were prepared at known concentrations from a stock solution and subjected to the same derivatization and extraction procedures. The detection limits were lower than $0.1 \mathrm{mg} / \mathrm{kg}$ for polyamines. Based on polyamine analysis in 10 runs of replicate samples, the inter-assay coefficient of variation $(\mathrm{CV})$ was $8.3-8.8 \%$.

\section{Data analysis}

Means and standard deviations of dietary polyamine content of Swedish dairy products as well as the coefficient of variation of polyamines were calculated using SPSS version 17.0, 2008, SPSS Inc.

\section{Results}

\section{Polyamine in foods from the database}

The literature search for any published values for putrescine, spermidine, and spermine provided data on polyamine content for more than 250 foods. The contents of polyamines in these foods are shown in Table 1 with references $(2,4-6,15-17,22,23,31-33,37-56)$. Fruits (like oranges), fruit juices (orange juice and grapefruit juice), sauerkraut, cheddar cheeses, cod roe, soy sauce, and soy miso were high in putrescine. Spermidine content was high in dry soy bean, chicken liver, green peas, corn, shell fish, and blue cheese. A high content of spermine was found in most of the meat products (like sausages, pork, chicken, and turkey), some vegetables (like pumpkin), and cheese. This polyamine, on the other hand, was found in low quantities in other types of foods and frequently reported as not detected.

Based on the Dietist XP and portion size, the top 12 foods having the highest amounts of each polyamine in $\mathrm{mg}$ per portion were listed in Table 2. Polyamine contents according to food groups are shown in Fig. 1. The group of cheese was the highest source of spermidine, while the fruits group was the highest in putrescine. Both food groups were the most important sources of total polyamine. 


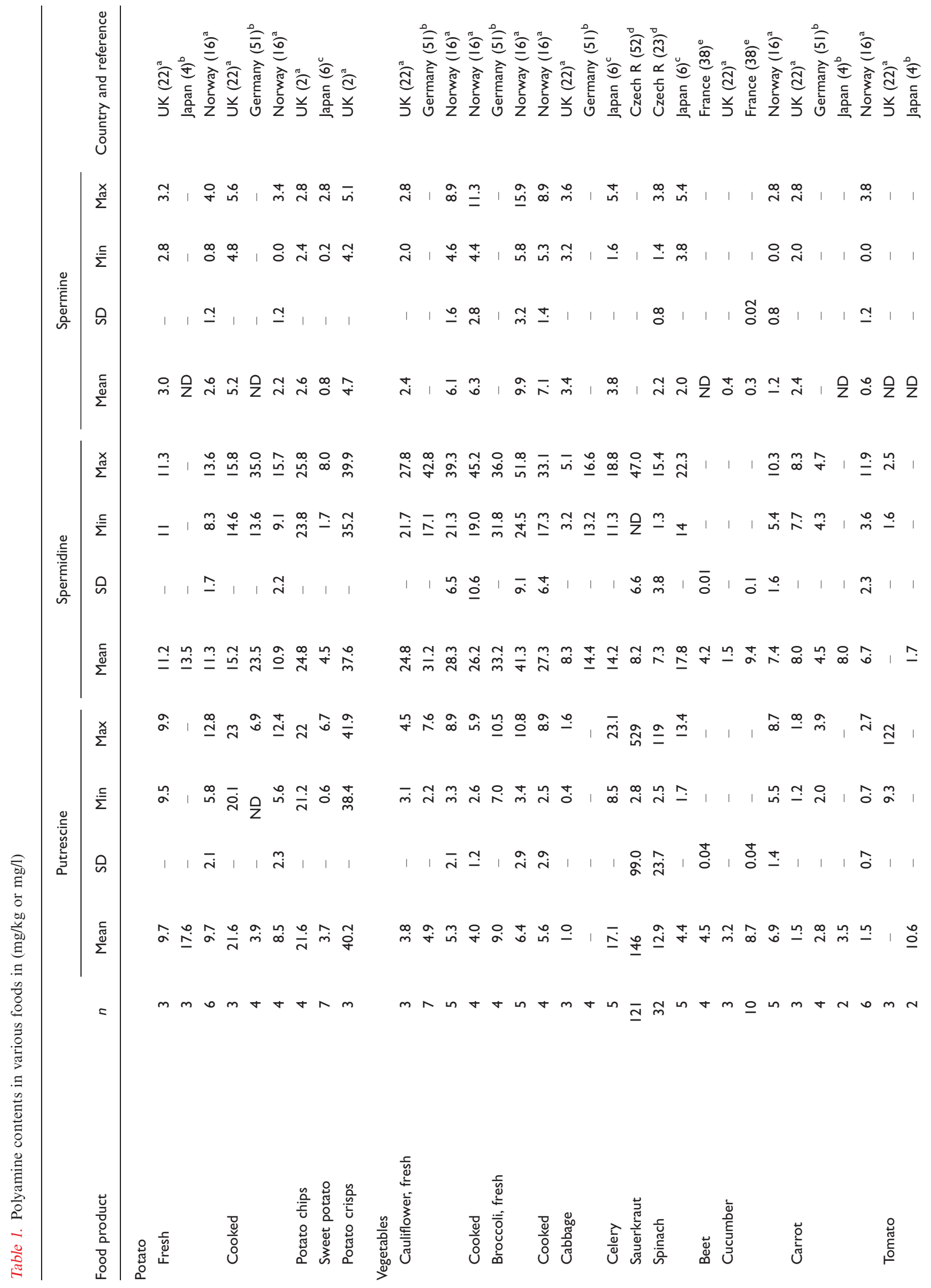




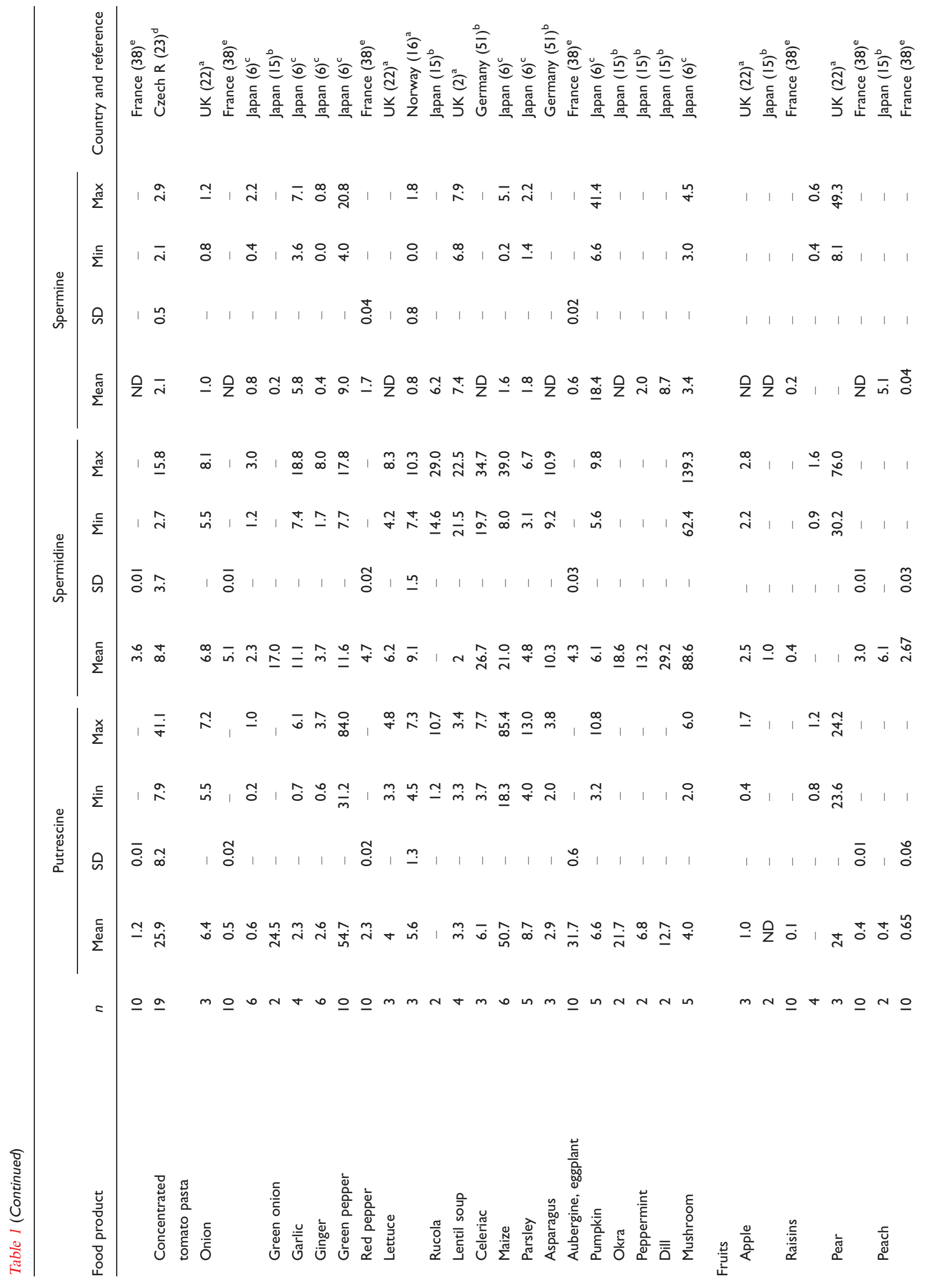




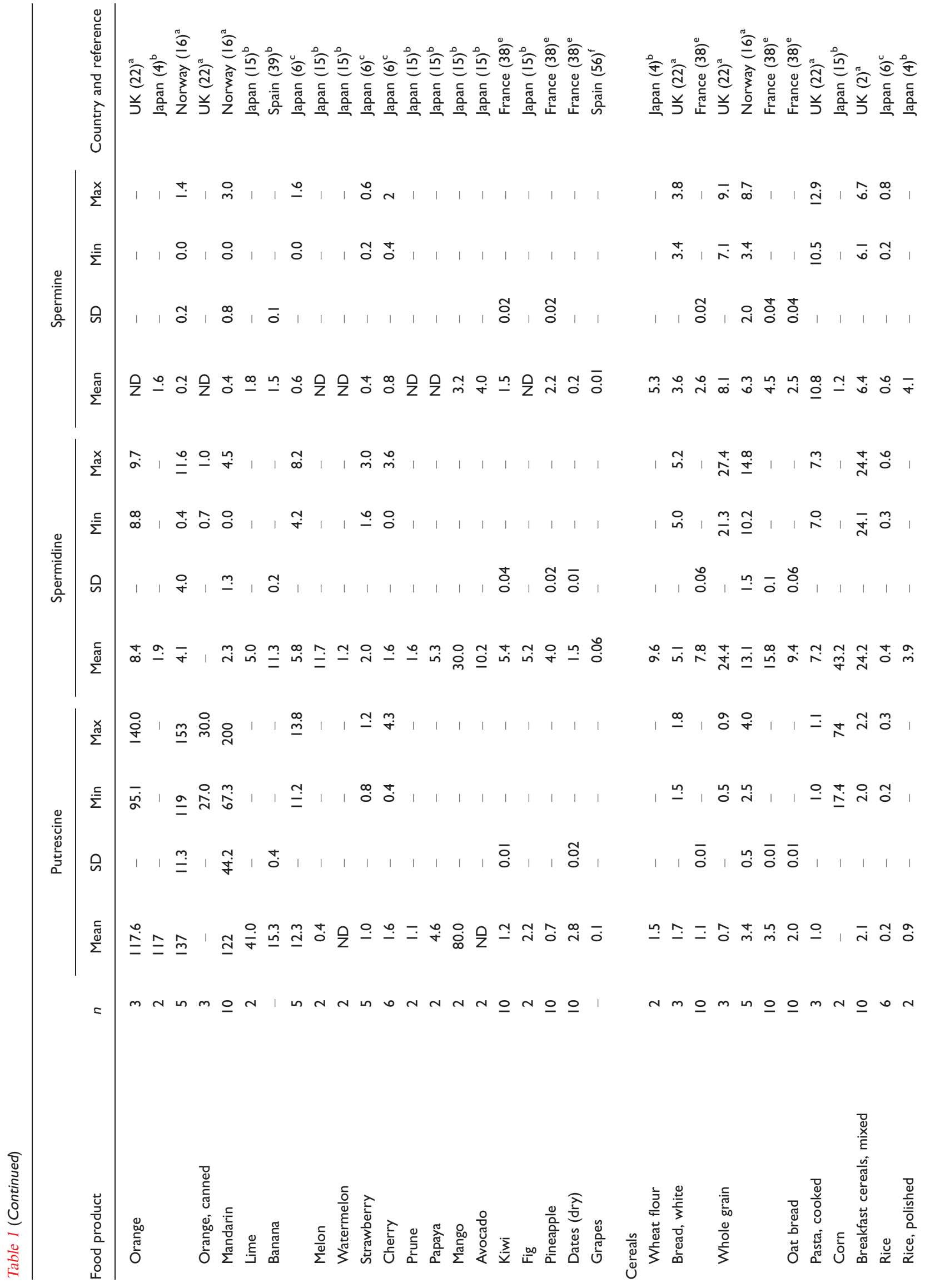




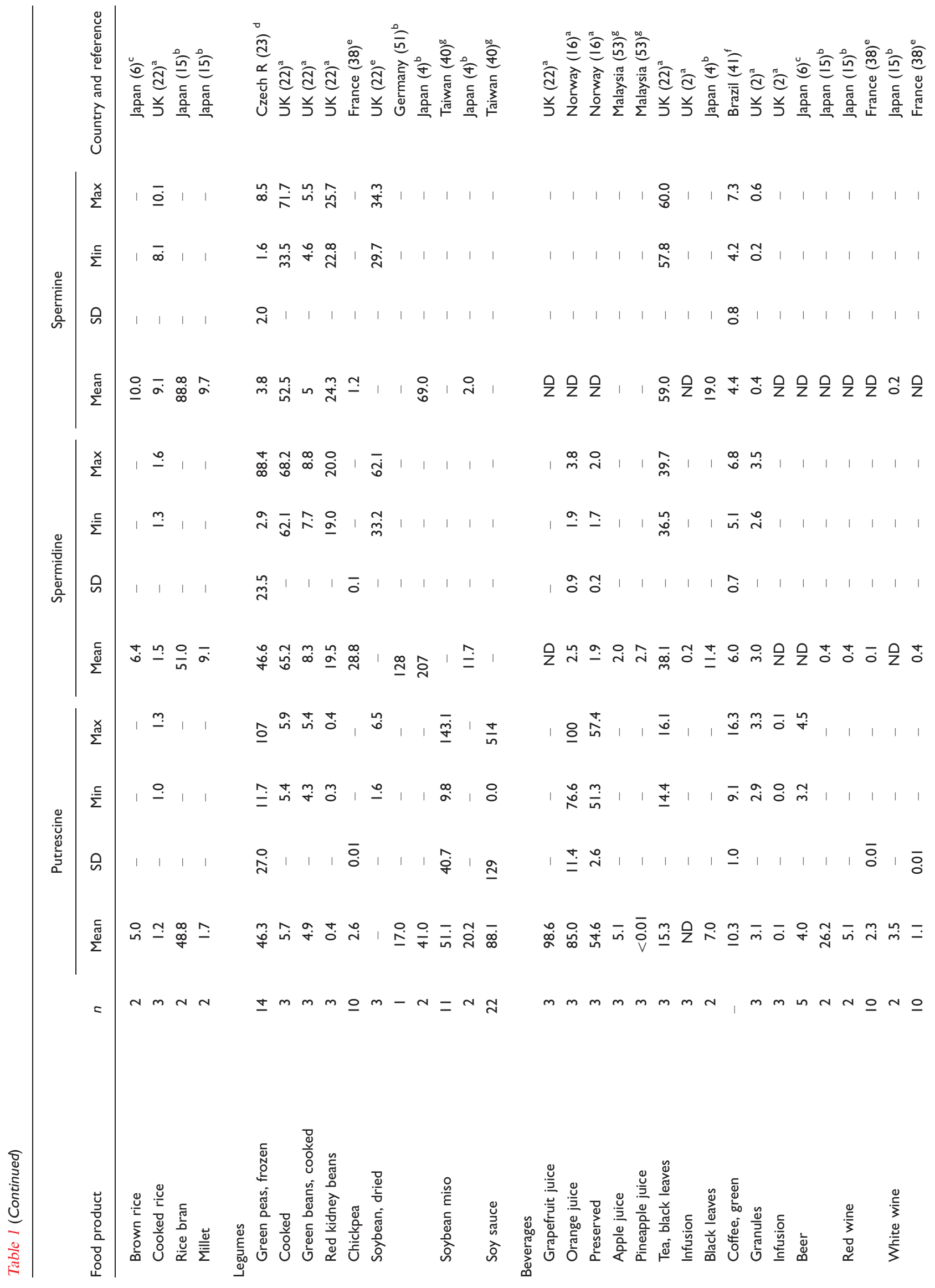




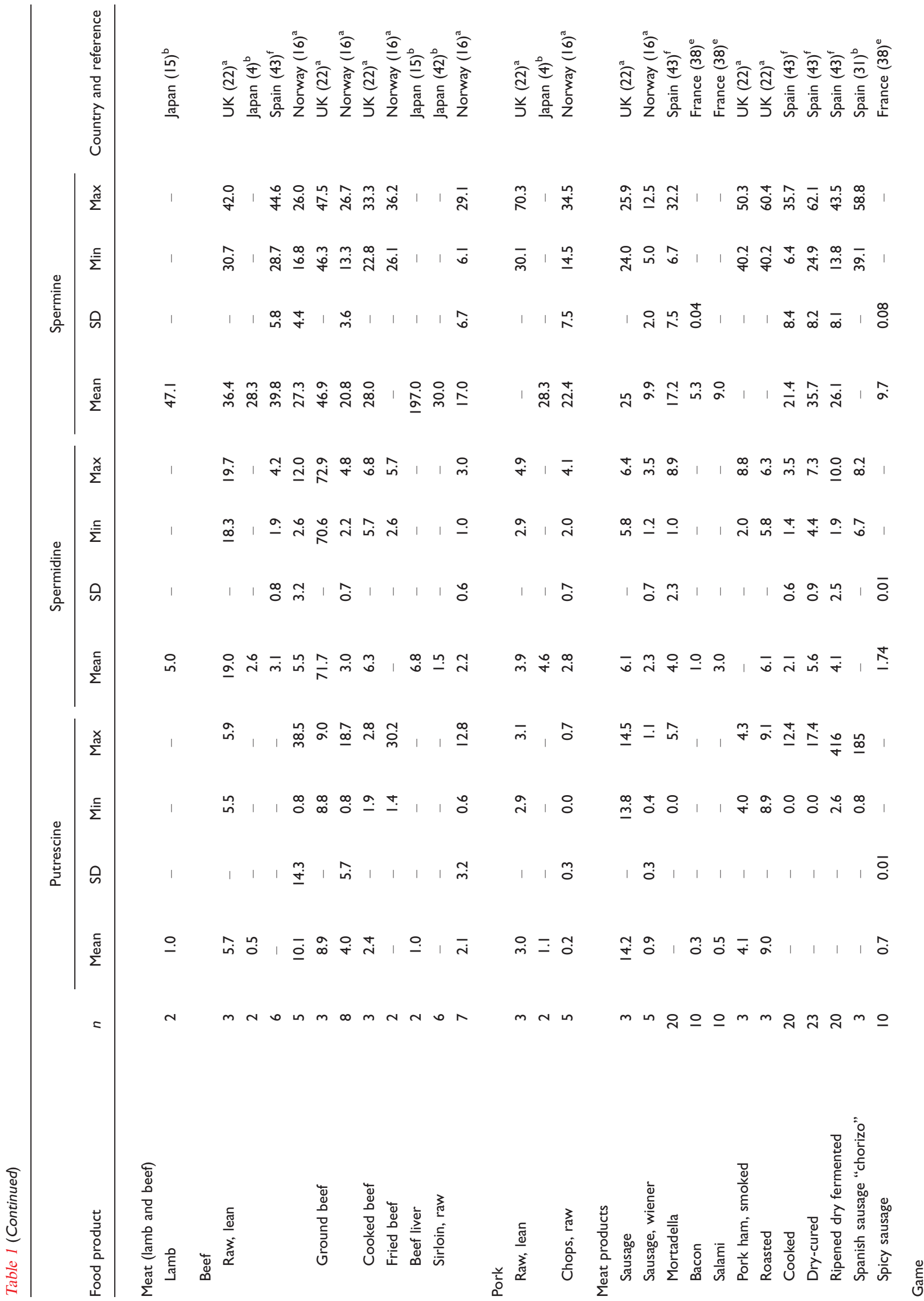




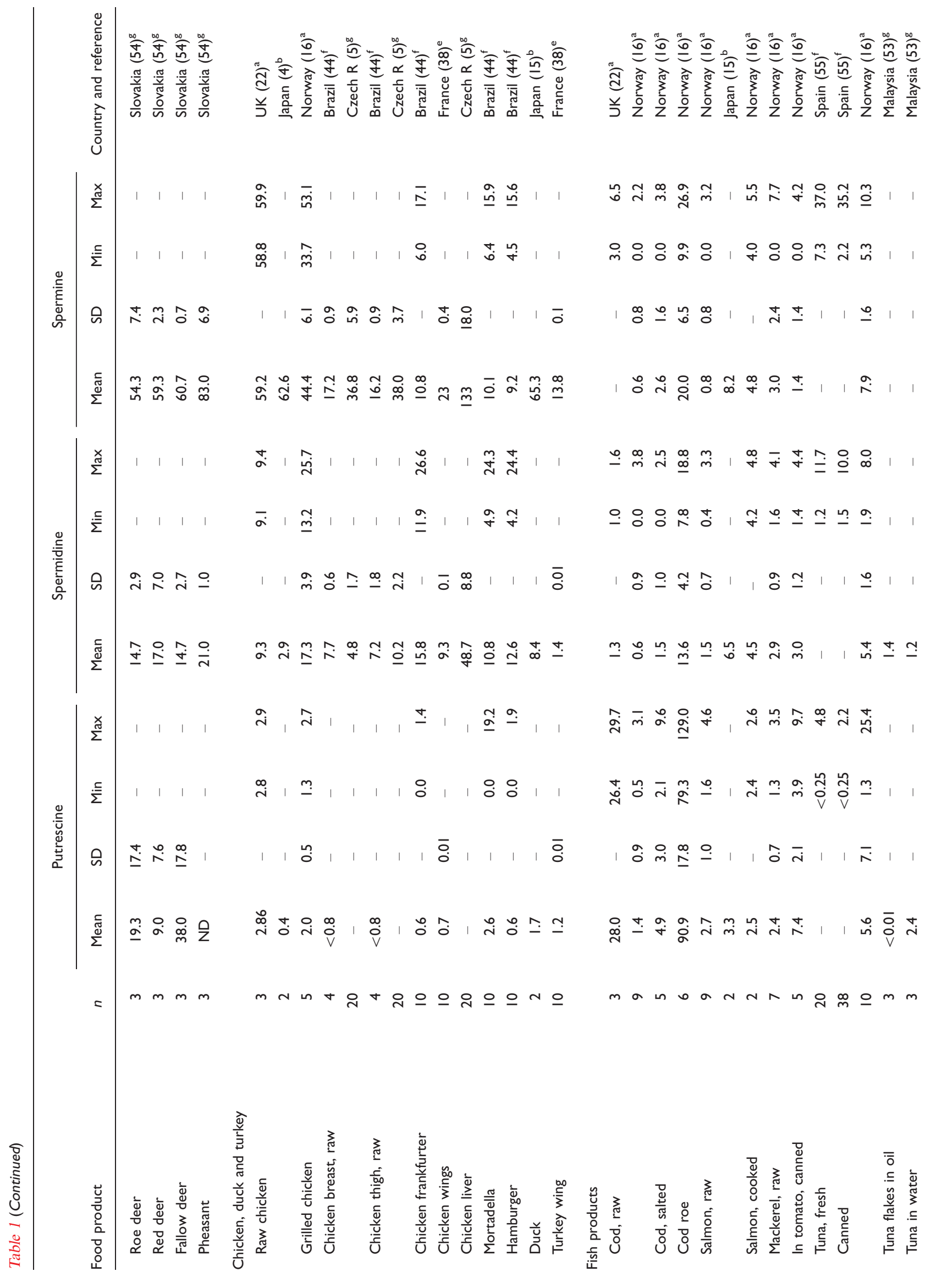




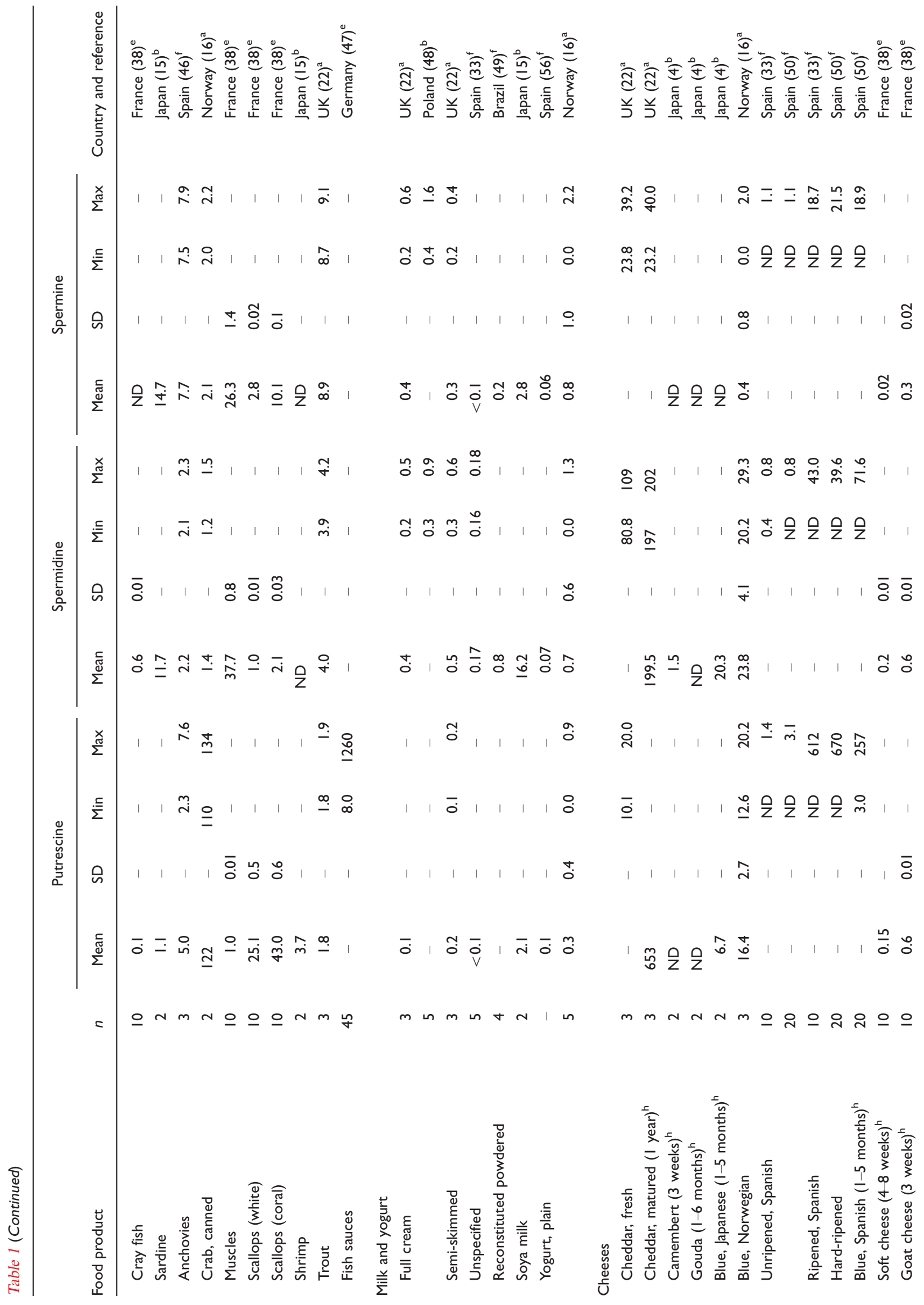




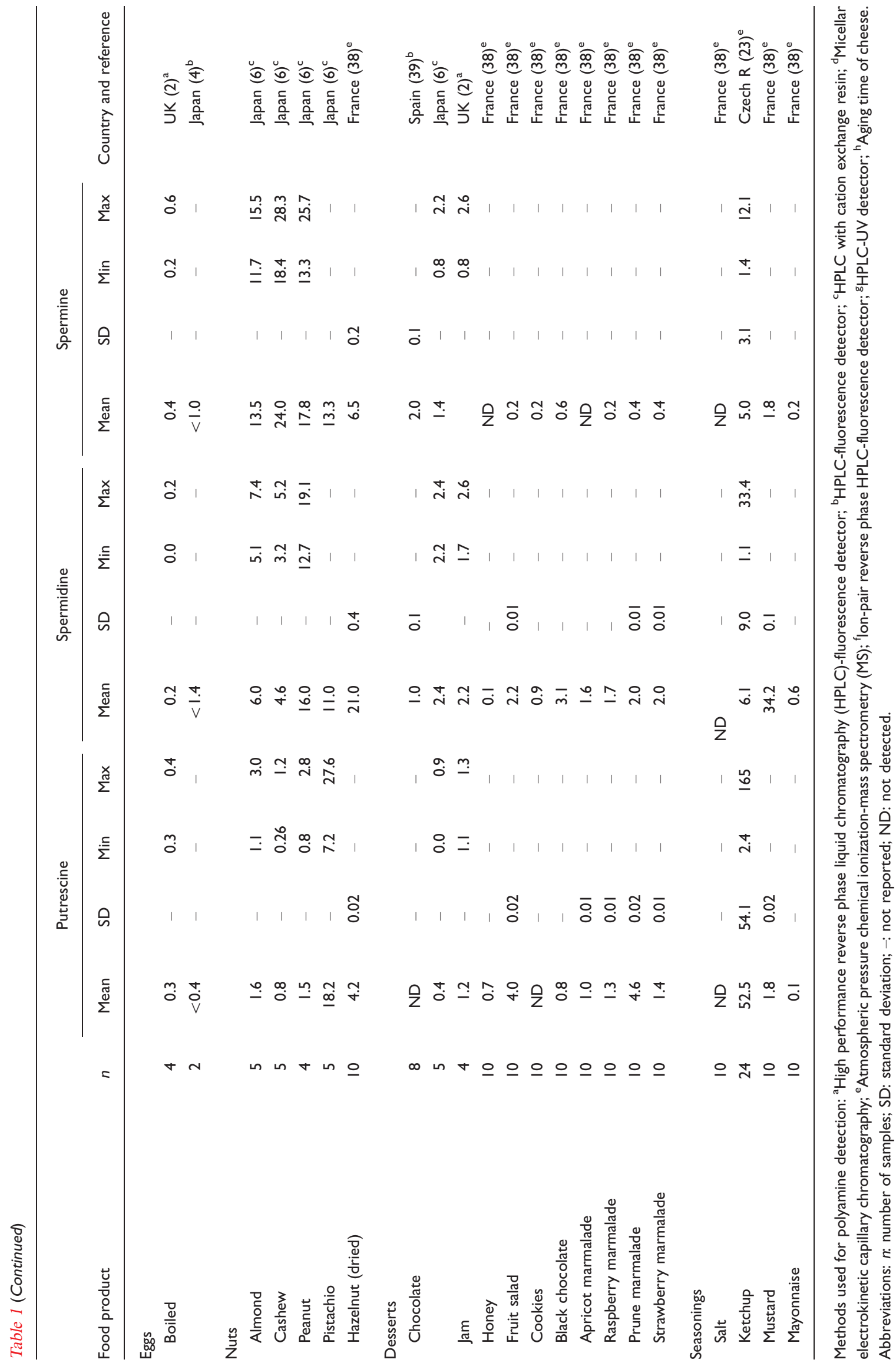


Table 2. Top 12 food sources of polyamines ( $\mathrm{mg} /$ portion) in the Dietist XP

\begin{tabular}{|c|c|c|}
\hline Polyamine/food item & Portions in grams & Polyamines $\mathrm{mg} /$ portion \\
\hline \multicolumn{3}{|l|}{ Putrescine } \\
\hline Grapefruit juice, fresh & 200 & 19.6 \\
\hline Orange juice & 200 & 17.0 \\
\hline Sauerkraut & 80 & 14.6 \\
\hline Orange & 110 & 14.0 \\
\hline Crab, conserved & 75 & 9.2 \\
\hline Maize & 100 & 5.1 \\
\hline Peas, green & 100 & 4.6 \\
\hline Pear & 100 & 3.0 \\
\hline Soybean, cooked & 190 & 1.70 \\
\hline Potato, cooked & 150 & 1.68 \\
\hline Paprika, green & 30 & 1.64 \\
\hline Soy sauce & 18 & 1.60 \\
\hline \multicolumn{3}{|l|}{ Spermidine } \\
\hline Soybean, cooked & 190 & 9.7 \\
\hline Peas, green & 140 & 9.1 \\
\hline Pear & 125 & 6.6 \\
\hline Lentil soup & 250 & 5.5 \\
\hline Mushroom & 50 & 4.4 \\
\hline Red beans & 190 & 3.7 \\
\hline Broccoli & 100 & 3.6 \\
\hline Cauliflower & 100 & 3.0 \\
\hline Chicken, steak & 125 & 2.2 \\
\hline Popcorn & 50 & 2.1 \\
\hline Cheese & 20 & 2.0 \\
\hline Potato, cooked & 150 & 1.8 \\
\hline \multicolumn{3}{|l|}{ Spermine } \\
\hline Liver (cow) & 125 & 19.7 \\
\hline Green peas & 140 & 7.3 \\
\hline Pork, ham & 125 & 6.3 \\
\hline Chicken & 125 & 5.6 \\
\hline Soybean, cooked & 190 & 4.0 \\
\hline Beef steak & 125 & 3.9 \\
\hline Pork & 125 & 3.8 \\
\hline Pear & 125 & 3.5 \\
\hline Cheeses & 100 & 3.0 \\
\hline Tuna fish & 125 & 2.7 \\
\hline Chicken breast & 125 & 2.3 \\
\hline Lentil soup & 250 & 1.85 \\
\hline
\end{tabular}

\section{Polyamine analysis of dairy products}

Table 3 shows the results from our laboratory analysis of typical Swedish dairy foods. The low fat milk and sour milk had the highest total polyamine contents, whereas the yogurt had the lowest contents. Sour milk had the highest mean putrescine contents. Total polyamine level was higher in cheese with long maturation than other types. Unlike the milk, cheese that differs in fat percentage still had similar values of polyamines.

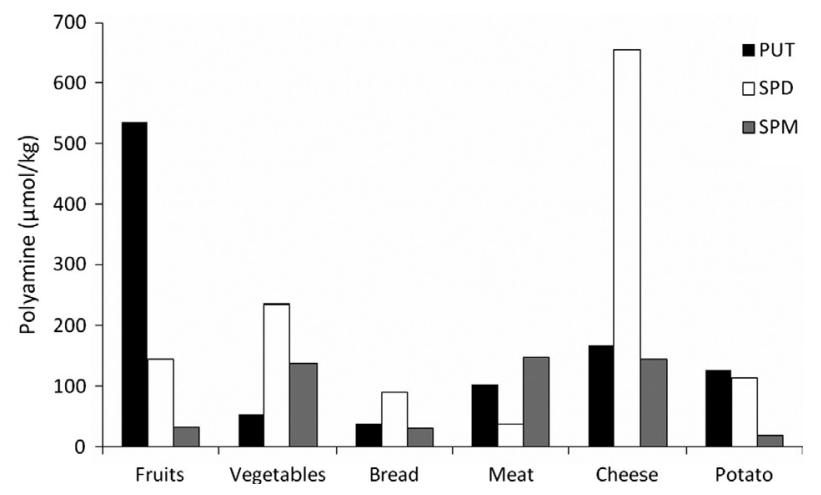

Fig. 1. Mean polyamine (putrescine, spermidine, and spermine) content in each food group according to the developed database. Number of foods included in each group: fruits (29), vegetables (46), bread (14), meat (55), cheese (19), and potato (6).

\section{Discussion}

Polyamine database

Polyamine content varies noticeably in food. Data obtained from different studies reflect this variation. Using different techniques for measuring polyamines, in addition to the other influencing factors such as storage periods and conditions as well as cooking method, might also play a role in affecting the resulting database $(5,57)$. These different analytical methods showed some variation in polyamine contents in the same type of food. As shown in Table 1, putrescine in tomato varied from 1.2 to $122 \mathrm{mg} / \mathrm{kg}$ in three different studies $(4,22,38)$. In addition, putrescine in sausage was another example of this variation not only between studies that have even used the same method $(16,22)$ but also varied in the same study. For example, the large variation in putrescine content in fermented Spanish sausage is explained by the metabolism of fermentative bacteria and the period of fermentation process (43). Similarly, the tremendous variation in putrescine concentration in 121 samples of sauerkraut $(2.8-529 \mathrm{mg} / \mathrm{kg})$ may be explained by the different microflora during spontaneous fermentation (52). However, values for polyamines in the main food sources such as putrescine in oranges, spermidine in Japanese and Norwegian blue cheese, and spermine in raw lean beef were similar across studies $(4,6,16,22)$.

Fermented soy bean has been reported to have considerable amounts of polyamines. Polyamines in fermented soy bean are considered to be formed by the action of lactic micro flora that is active during the fermentation process (58-60).

In Table 2, cooked soy bean and cow's liver were the highest sources for spermidine and spermine, respectively, whereas for putrescine, sauerkraut appeared to be the third important source due to its lower portion size than grape fruits and oranges. These figures differ slightly 


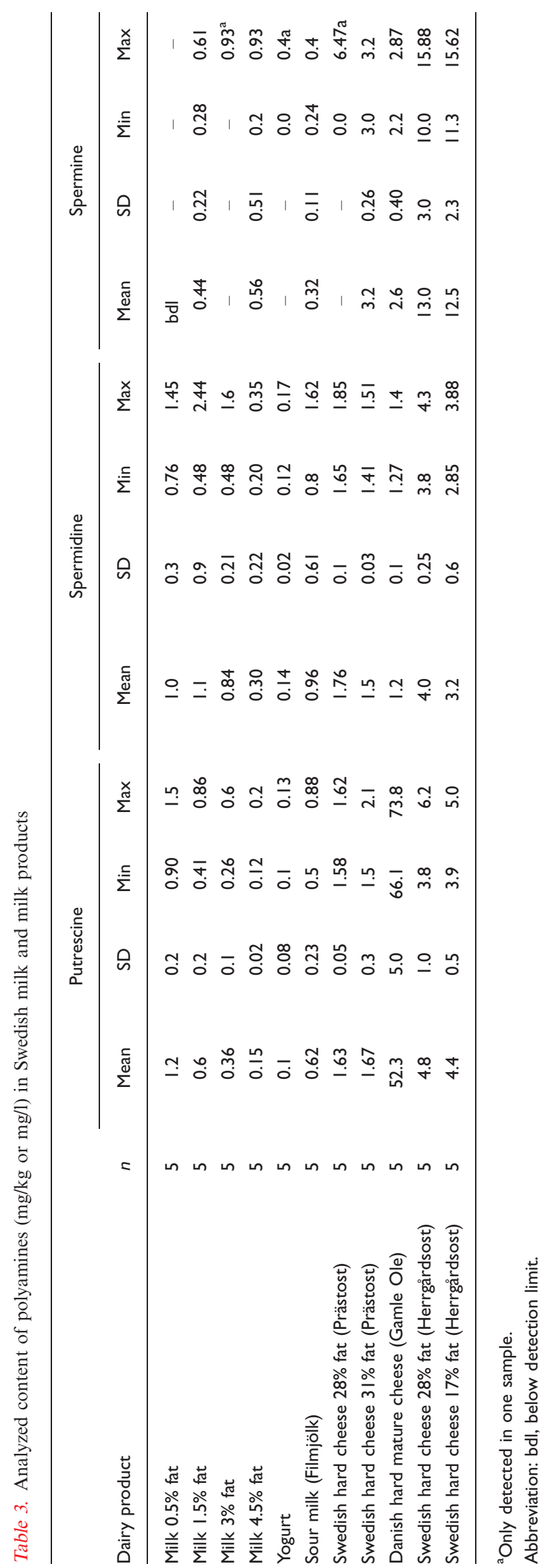

from an American study where the top 10 foods with the highest polyamine contents were reported (26). Fresh or canned corn and green pea soup had the highest spermidine and spermine, respectively. This difference could be due to the fact that the mean values for each polyamine that were estimated in their food database differ slightly from ours and also due to difference in portion size estimates.

\section{Polyamine in dairy products}

Our laboratory results showed that Swedish cheese has higher polyamine content than milk. This is natural since cheese is essentially concentrated milk. Such a finding was in accordance with other hard cheese variants from literature $(16,33,50)$. The values for the mean total polyamine content, particularly putrescine and spermine in selected Swedish mature cheese were comparable to those in Spanish hard cheese (33). However, there were still reported variations in polyamine content in cheese between the studies $(4,16,33,38)$. This could be due to differences in the fermentation process and the starter culture employed in the cheese production $(16,58,61)$.

As reported for other studies on polyamine contents in cheeses $(4,16,22,50)$, the putrescine content varied between matured and unmatured cheese. The high content of putrescine in matured cheese is expected to be a result of the fermentation process. A previous study showed that lactobacillus acidophilus, Streptococcus faecium, and Lactobacillus casei, which were isolated from fermented cheese, were responsible for putrescine production (61). In addition, using pasteurized milk and mesophilic or thermophilic starter bacteria favored the formation of polyamine in cheese (60). Spermine and spermidine contents were higher in Herrgårdsost, a common Swedish short maturation cheese, independent of fat content (17 and 28\% fat). Herrgårdsost is made by introducing bacterial starters to pasteurized milk including lactic acid bacteria, which acidify the milk. This bacterial process besides the short maturation would explain the higher putrescine contents in Herrgårdost than in Prästost. In Prästost with $28 \%$ fat, spermine was detected only in one sample out of five. This polyamine was twice as much the content in $31 \%$ Prästost. This difference in spermine within the same type of cheese can be explained by polyamines variability not only between different types of cheese but also within the same type, which could be attributed to difference in the manufacturing process (50).

In milk, the lower the fat content, the higher the polyamine content. It has been previously reported that high fats and protein proportions might hinder polyamine extraction (33). Therefore, such effect has been considered in our method by careful polyamine extraction. Moreover, putrescine concentration in milk, particularly low fat milk, was about 10 times higher than the 
concentrations that were reported previously (22). This variation might be explained by the effect of milk processing on the levels of polyamines; however, when non-homogenized high fat milk was analyzed for its polyamine content, lower polyamine values were detected. Another explanation for the variation within and between studies could be the effect of seasonal variations on milk composition, storage conditions, and environmental influences and the different techniques used for polyamine determination $(3,48)$. The very low polyamine content in yogurt was in agreement with the contents reported from other studies $(16,56)$.

\section{Limitations of the study}

We did not have a large sample size when it comes to our own analyses of dairy products. However, the dairy products selected were the most commonly purchased brands in Sweden and the variation in the results from our chosen sensitive analytical method was minor.

\section{Conclusions}

Information on polyamines content in foods has been required due to the role of these dietary compounds in health and diseases. Therefore, a database on polyamines in foods was developed to provide an estimate for the mean total and individual polyamine intake, based on food intake derived from dietary surveys.

The variation in polyamines content is considerable and may be related to raw material, manufacturing and storage processes, different methodological approaches, and seasonal variation. This variation between different studies, besides the one even within the same type of food, underlines the need for caution when interpreting the results from literature. Reasons for the almost 10 -fold variation in polyamine content demonstrated in milk samples with different fat content needs further investigation.

\section{Conflict of interest and funding}

The authors have not received any funding or benefits from any industry to conduct this study.

\section{References}

1. National Food Administration. http://www.slv.se/en-gb/Group1/ Food-and-Nutrition/The-Food-Database/ [cited 6 December 2010].

2. Bardocz S, Duguid TJ, Brown DS, Grant G, Pusztai A, White A, et al. The importance of dietary polyamines in cell regeneration and growth. Br J Nutr 1995; 73: 819-28.

3. Loser C. Polyamines in human and animal milk. Br J Nutr 2000; 84: S55-S58

4. Okamoto A, Sugi E, Koizumi Y, Yanagida F, Udaka S. Polyamine content of ordinary foodstuffs and various fermented foods. Biosci Biotechnol Biochem 1997; 61: 1582-4.

5. Kozova M, Kalac P, Pelikanova T. Contents of biologically active polyamines in chicken meat, liver, heart and skin after slaughter and their changes during meat storage and cooking. Food Chem 2009; 116: 419-25.

6. Nishibori N, Fujihara S, Akatuki T. Amounts of polyamines in foods in Japan and intake by Japanese. Food Chem 2006; 100: 491-7.

7. Moinard C, Cynober L, de Bandt JP. Polyamines: metabolism and implications in human diseases. Clin Nutr 2005; 24: 184-97.

8. Ferioli ME, Pirona L, Pinotti O. Prolactin and polyamine catabolism: specific effect on polyamine oxidase activity in rat thymus. Mol Cell Endocrinol 2000; 165: 51-6.

9. Hoet PH, Nemery B. Polyamines in the lung: polyamine uptake and polyamine-linked pathological or toxicological conditions. Am J Physiol Lung Cell Mol Physiol 2000; 278: 417-33.

10. Dandrifosse G, Peulen O, El Khefif N, Deloyer P, Dandrifosse AC, Grandfils C. Are milk polyamines preventive agents against food allergy? Proc Nutr Soc 2000; 59: 81-6.

11. Gugliucci A, Menini T. The polyamines spermine and spermidine protect proteins from structural and functional damage by AGE precursors: a new role for old molecules? Life Sci 2003; 72: 2603-16.

12. Tabor CW, Tabor H. Polyamines. Annu Rev Biochem 1984; 53 : 749-90.

13. Loser C, Folsch UR. Importance of various intracellular regulatory mechanisms of polyamine metabolism in camostate-induced pancreatic growth in rats. Digestion 1993; 54: 213-23.

14. Bardocz S. Polyamines in food and their consequences for food quality and human health. Trends Food Sci Technol 1995; 6: 341-6.

15. Nishimura K, Shiina R, Kashiwagi K, Igarashi K. Decrease in polyamines with aging and their ingestion from food and drink. J Biochem (Tokyo, Japan) 2006; 139: 81-90.

16. Eliassen KA, Reistad R, Risoen U, Ronning HF. Dietary polyamines. Food Chem 2002; 78: 273-80.

17. Larque E, Sabater-Molina M, Zamora S. Biological significance of dietary polyamines. Nutrition (New York) 2007; 23: 87-95.

18. Dufour C, Dandrifosse G, Forget P, Vermesse F, Romain N, Lepoint P. Spermine and spermidine induce intestinal maturation in the rat. Gastroenterology 1988; 95: 112-6.

19. Deloyer P, Peulen O, Dandrifosse G. Dietary polyamines and non-neoplastic growth and disease. Eur J Gastroenterol Hepatol 2001; 13: 1027-32.

20. Das R, Kanungo MS. Activity and modulation of ornithine decarboxylase and concentrations of polyamines in various tissues of rats as a function of age. Exp Gerontol 1982; 17: 95-103.

21. Gerner EW, Meyskens FL Jr. Polyamines and cancer: old molecules, new understanding. Nat Rev Cancer 2004; 4: 781-92.

22. Bardócz S, Grant G, Brown DS, Ralph A, Pusztai A. Polyamines in food - implications for growth and health. J Nutr Biochem 1993; 4: 66-71.

23. Kalac P, Svecova S, Pelikanova T. Levels of biogenic amines in typical vegetable products. Food Chem 2002; 77: 349-51.

24. Bauza T, Kelly MT, Blaise A. Study of polyamines and their precursor amino acids in Grenache noir and Syrah grapes and wine of the Rhone Valley. Food Chem 2007; 105: 405-13.

25. Friedman R, Altman A, Levin N. The effect of salt stress on polyamine biosynthesis and content in mung bean plants and in halophytes. Physiol Plant 1989; 76: 295-302.

26. Zoumas-Morse C, Rock CL, Quintana EL, Neuhouser ML, Gerner EW, Meyskens FL Jr. Development of a polyamine database for assessing dietary intake. J Am Diet Assoc 2007; 107: 1024-7.

27. Ralf A, Englyst K. Polyamine content of human diet. In: Bardocz S, White A, eds. Polyamines in health and nutrition. Norwell, MA: Kluwer Academic Publisher; 1999. pp. 123-37. 
28. Lagishetty CV, Naik SR. Polyamines: potential anti-inflammatory agents and their possible mechanism of action. Indian J Pharmacol 2008; 40: 121-5.

29. Krausova P, Kalac P, Krizek M, Pelikanova T. Content of polyamines in beef and pork after animal slaughtering. Eur Food Res Technol 2006; 223: 321-4.

30. Kalac P. Biologically active polyamines in beef, pork and meat products: a review. Meat Science 2006; 73: 1-11.

31. Ruiz-Capillas C, Jimenez-Colmenero F. Biogenic amine content in Spanish retail market meat products treated with protective atmosphere and high pressure. Eur Food Res Technol 2004; 218: $237-41$

32. Kalac P, Krizek M, Pelikanova T, Langova M, Veskrna O. Contents of polyamines in selected foods. Food Chem 2004; 90: $561-4$.

33. Novella-Rodriguez S, Veciana-Nogues MT, Vidal-Carou MC. Biogenic amines and polyamines in milks and cheeses by ionpair high performance liquid chromatography. J Agric Food Chem 2000; 48: 5117-23.

34. Vale SR, Gloria MBA. Determination of biogenic amines in cheese. J AOAC Int 1997; 80: 1006-12.

35. Taibi G, Schiavo MR. Simple high-performance liquid chromatographic assay for polyamines and their monoacetyl derivatives. J Chromatogr 1993; 614: 153-8.

36. Mei YH. A sensitive and fast method for the determination of polyamines in biological samples - benzoyl chloride precolumn derivatization high-performance liquid-chromatography. J Liq Chromatogr 1994; 17: 2413-8.

37. Bardocz S. The role of dietary polyamines. Eur J Clin Nutr 1993; 47: 683-90.

38. Cipolla BG, Havouis R, Moulinoux JP. Polyamine contents in current foods: a basis for polyamine reduced diet and a study of its long term observance and tolerance in prostate carcinoma patients. Amino Acids 2007; 33: 203-12.

39. Lavizzari T, Teresa Veciana-Nogues M, Bover-Cid S, MarineFont A, Carmen Vidal-Carou M. Improved method for the determination of biogenic amines and polyamines in vegetable products by ion-pair high-performance liquid chromatography. J Chromatogr A 2006; 1129: 67-72.

40. Yen GC. Biogenic amines in foods. I. Determination of biogenic amines in fermented soybean foods by HPLC. Zhongguo Nongye Huaxue Huizhi 1986; 24: 211-27.

41. Cirilo MPG, Coelho AFS, Araujo CM, Goncalves FRB, Nogueira FD, Gloria MBA. Profile and levels of bioactive amines in green and roasted coffee. Food Chem 2003; 82: 397 402.

42. Yano Y, Kataho N, Watanabe M, Nakamura T, Asano Y. Changes in the concentration of biogenic amines and application of tyramine sensor during storage of beef. Food Chem 1995; 54: 155-9.

43. Hernandez-Jover T, Izquierdo-Pulido M, Veciana-Nogues MT, Marine-Font A, Vidal-Carou MC. Biogenic amine and polyamine contents in meat and meat products. J Agric Food Chem 1997; 45: 2098-102.

44. Silva CMG, Gloria MBA. Bioactive amines in chicken breast and thigh after slaughter and during storage at $4 \pm 1^{\circ} \mathrm{C}$ and in chicken-based meat products. Food Chem 2002; 78: 241-8.

45. Kalac P, Krausová P. A review of dietary polyamines: formation, implications for growth and health and occurrence in foods. Food Chem 2005; 90: 219-30.

46. Veciana-Nogues MT, Marine-Font A, Vidal-Carou MC. Changes in biogenic amines during the storage of Mediterra- nean anchovies immersed in oil. J Agric Food Chem 1997; 45: 1385-9.

47. Stute R, Petridis K, Steinhart H, Biernoth G. Biogenic amines in fish and soy sauces. Eur Food Res Technol 2002; 215: 101-7.

48. Motyl T, Ploszaj T, Wojtasik A, Kukulska W, Podgurniak M. Polyamines in cow's and sow's milk. Comp Biochem Physiol B Biochem Mol Biol 1995; 111: 427-33.

49. Santos WC, Souza MR, Cerqueira MMOP, Gloria MBA. Bioactive amines formation in milk by Lactococcus in the presence or not of rennet and $\mathrm{NaCl}$ at 20 and $32^{\circ} \mathrm{C}$. Food Chem 2003; 81: 595-606.

50. Novella-Rodriguez S, Veciana-Nogues MT, Izquierdo-Pulido M, Vidal-Carou MC. Distribution of biogenic amines and polyamines in cheese. J Food Sci 2003; 68: 750-5.

51. Ziegler W, Hahn M, Wallnöfer PR. Changes in biogenic amine contents during processing of several plant foods [in German]. Deutsche Lebensmittel-Rundschau 1994; 90: 108-12.

52. Kalac P, Spicka J, Krizek M, Steidlova S, Pelikanova T. Concentrations of seven biogenic amines in sauerkraut. Food Chem 1999; 67: 275-80.

53. Saaid M, Saad B, Hashim NH, Ali ASM, Saleh MI. Determination of biogenic amines in selected Malaysian food. Food Chem 2009; 113: 1356-62.

54. Dicáková Z, Paulsen P, Bystrický P, Sokol J, Laczkóová S. Determination of biogenic amines and free amino acids in game meat during storage. In: Bystrický P, Nagy J, Máté D, eds. Hygiena Alimentorum XXIV, Slovakia, trbské Pleso. Košice, Slovakia: University of Veterinary Medicine; 2003. pp. 9799.

55. Veciana-Nogués MT, Mariné-Font A, Vidal-Carou MC. Biogenic amines in fresh and canned tuna. Effects of canning on biogenic amine contents. J Agric Food Chem 1997; 45: 4324-8.

56. Farriol M, Venereo Y, Orta X, Company C, Gomez P, Delgado $\mathrm{G}$, et al. Ingestion of antioxidants and polyamines in patients with severe burns [in Spanish]. Nutr Hosp 2004; 19: 300-4.

57. Rodriguez S, Lopez B, Chaves AR. Effect of different treatments on the evolution of polyamines during refrigerated storage of eggplants. J Agric Food Chem 2001; 49: 4700-5.

58. Kirschbaum J, Rebscher K, Brückner H. Liquid chromatographic determination of biogenic amines in fermented foods after derivatization with 3,5-dinitrobenzoyl chloride. J Chromatogr A 2000; 881: 517-30.

59. Stratton JE, Hutkins RW, Taylor SL. Biogenic amines in cheese and other fermented food: a review. J Food Protec 1991; 54: $460-70$.

60. Gennaro MC, Gianotti V, Marengo E, Pattono D, Turi RM. A chemometric investigation of the effect of the cheese-making process on contents of biogenic amines in a semi-hard Italian cheese (Toma). Food Chem 2003; 82: 545-51.

61. Shalaby AR. Significance of biogenic amines to food safety and human health. Food Res Int 1996; 29: 675-90.

\footnotetext{
*Mohamed Atiya Ali

Unit for Public health Nutrition

Department of Biosciences and Nutrition

NOVUM

SE-14I 83 Huddinge, Sweden

Tel: +460852481152

Fax: +46086083350

Email: mohamed.ali@ki.se
} 